Pathologe 2008 · [Suppl 2] 29:218-222

DOI 10.1007/s00292-008-1036-9

Online publiziert: 16. August 2008

(c) Springer Medizin Verlag 2008

\author{
L. Morawietz ${ }^{1} \cdot$ G. Fernahl ${ }^{1} \cdot$ V. Krenn ${ }^{2}$ \\ ${ }^{1}$ Institut für Pathologie, Charité-Universitätsmedizin Berlin \\ ${ }^{2}$ Institut für Pathologie, Wissenschaftspark Trier
}

\section{Differenzialdiagnostik der Synovialitis}

Von der Synovialmembran ist die so genannte Neosynovialis abzugrenzen, welche sich um ein Gelenk bildet, das durch eine Totalendoprothese ersetzt wurde. Diese wiederum unterscheidet sich von der so genannten periprothetischen Membran, einem bindegewebigen Saum, der zwischen einem Implantat und seinem knöchernen Lager zu finden ist. Für die histopathologische Klassifikation der Neosynovialis und der periprothetischen Membran empfiehlt sich ein System, welches 4 Typen (abriebinduziert, infektiös, Mischtyp, Indifferenztyp) unterscheidet. Dies soll hier nicht weiter erläutert werden, es fand jedoch Erwähnung, logische Veränderung zu werten wäre.

mittleren Kapillardichte aufliegt. In letzterem wird die Synovia, auch als Gelenkflüssigkeit bezeichnet, gebildet und resorbiert. Physiologischerweise kann die Synovialmembran Falten (Plicae) und Zotten (Villi) aufweisen, ohne dass dies als patho-

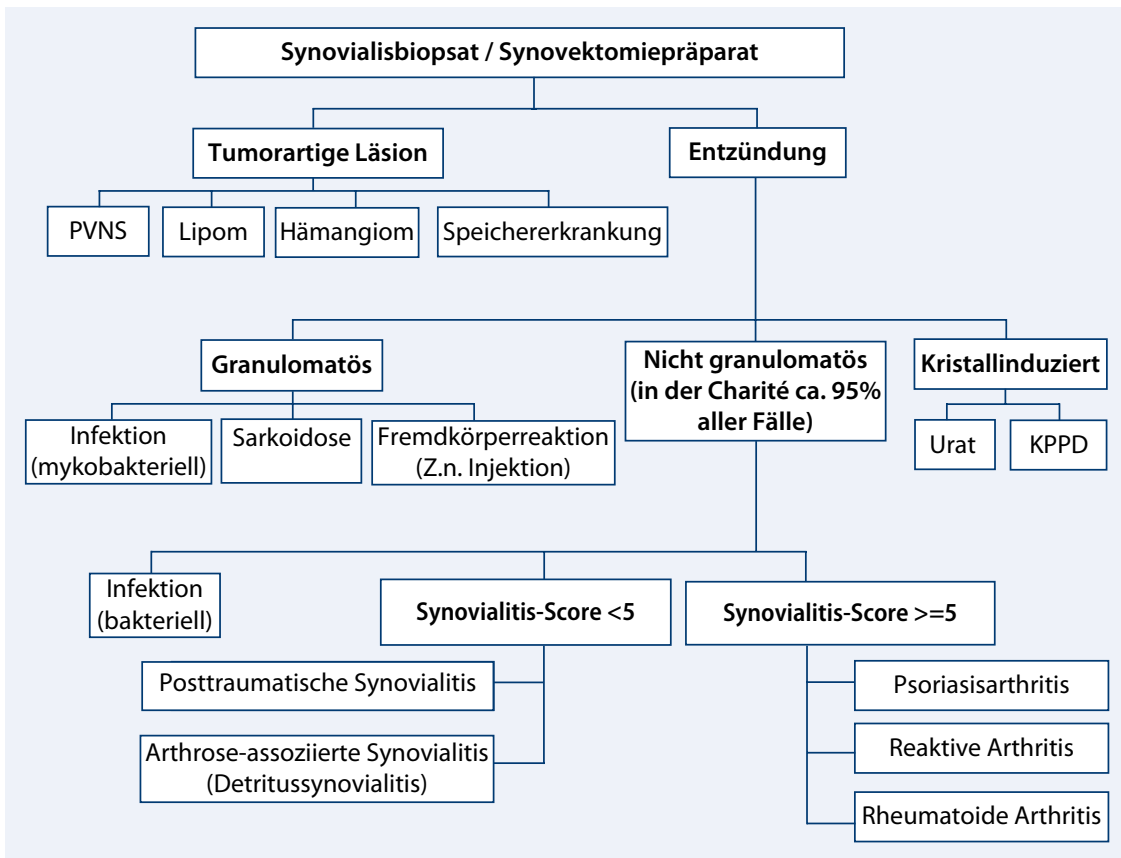

Abb. $1 \Delta$ Flussdiagramm zur standardisierten histopathologischen Beurteilung von Synovialisbiopsaten bzw. Synovektomiepräparaten (KPPD Kalziumpyrophosphatdihydrat) da klinischerseits auf den Probenbegleitscheinen gelegentlich eine unscharfe Verwendung der unterschiedlichen Termini vorkommt, sodass beispielsweise unter der Probenbezeichnung „Synovialmembran" eine periprothetische Membran eingesandt wird [11].

\section{Vielfalt der Gelenkerkrankungen}

An den Gelenken manifestiert sich eine Vielzahl von Erkrankungen, die degenerativer, primär entzündlicher, metabolischer oder auch neoplastischer Natur sein können. Die meisten dieser Krankheiten verlaufen chronisch und gehen mit Schmerzen und Bewegungseinschränkungen einher. Das diagnostische Problem ist, dass sich nur ein kleinerer Teil dieser Erkrankungen durch spezifische histopathologische Veränderungen eindeutig identifizieren lässt, wie z. B. eine Tuberkulose, die Gicht oder der tenosynoviale Riesenzelltumor [10].

Die Diagnose der entzündlichen und der degenerativen Gelenkerkrankungen wird in erster Linie anhand klinischer, laborchemischer und radiologischer Kriterien gestellt. Lange Zeit wurden keine Biopsate aus den Gelenken gewonnen, obwohl die „American Rheumatism Association“ (ARA) in ihrer ursprünglichen Version der definierenden Kriterien der rheumatoiden Arthritis (RA, auch als primär chronische Polyarthritis bezeichnet) von 1958 auch histologische Aspekte einbezog. Diese wurden in die modifizierten Kriterien von 1987 jedoch nicht mehr aufgenommen. Grund dafür war weniger die Aussagekraft der Biopsate - die Spezifität wurde mit 95,0\% und die Exaktheit mit 
Abb. 2 a Uratkristalle (Pfeile) innerhalb von Riesenzellen; umgebend ein entzündliches Infiltrat aus Makrophagen und wenigen Lymphozyten und Granulozyten bei der Gicht (HE-Färbung, Vergr. 200:1). b Im Vergleich dazu enthält die Pseudogicht nahezu keine Entzündungszellen, und die KPPD-Depositionen (Pfeile) zeigen einen etwas graueren Farbton (HE-Färbung, Vergr. 100:1)
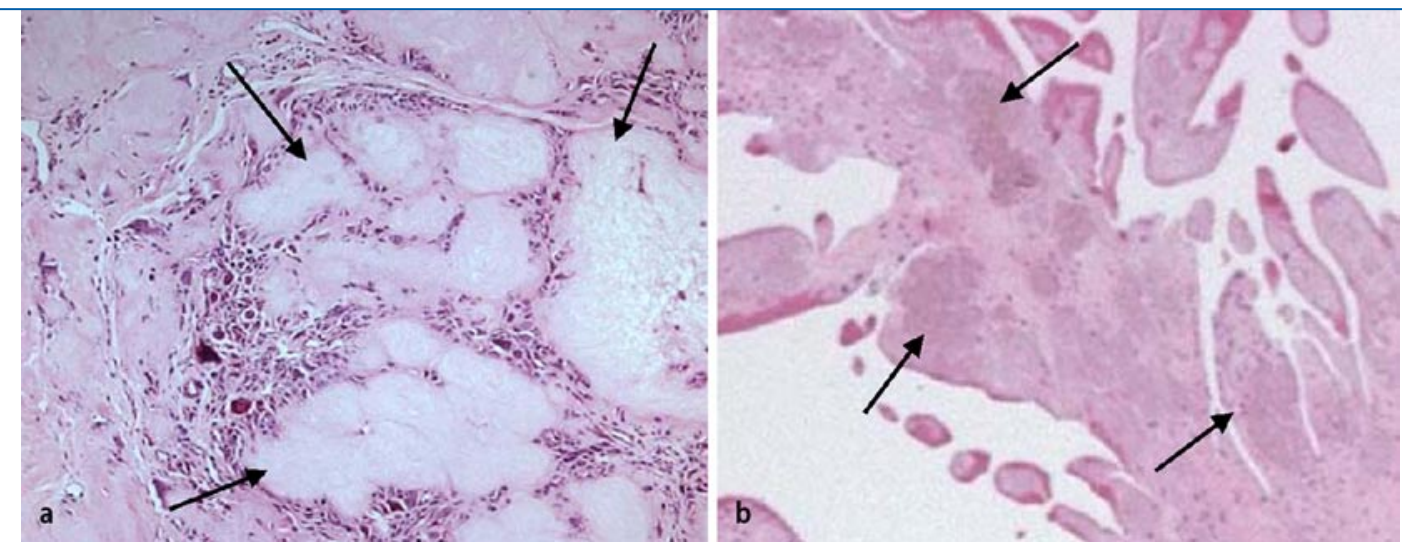

$68,7 \%$ angegeben - als vielmehr die Tatsache, dass eine Biopsie von niedergelassenen Rheumatologen nur selten durchgeführt wurde [2]. Dies hat sich inzwischen wieder geändert. Zur Befundsicherung oder zur Klärung uncharakteristischer Krankheitsbilder werden vermehrt Synovialisbiopsate gewonnen, und auch therapeutische Synovektomiepräparate werden zur histologischen Untersuchung übersandt [15].

Um den großen Teil der uncharakteristischen chronischen Gelenkerkrankungen standardisiert und aussagefähig bewerten zu können, wurde ein Gradierungssystem, der so genannte Synovialitis-Score erstellt, der sich auf die 3 wesentlichen morphologischen Aspekte der chronischen Synovialtis stützt $[4,5,6]$.

\section{Histopathologische Diagnostik von Synovialispräparaten}

Einen Überblick über die Vielfalt der Diagnosen und einen Anhaltspunkt für die differenzialdiagnostische Abklärung gibt der in - Abb. 1 dargestellte Algorithmus. Es sollte zunächst ausgeschlossen werden, dass eine tumorartige Veränderung vorliegt (1. Ebene). Entzündungen lassen sich ggf. als granulomatös, kristallinduziert oder uncharakteristisch identifizieren (2. Ebene). Ein Teil der uncharakteristischen Synovialitiden ist floride und muss mikrobiologisch weiter abgeklärt werden. Für die weitere Beurteilung der chronischen uncharakteristischen Synovialitiden empfiehlt sich die Anwendung des Synovialitis-Scores (3. Ebene).

\section{Gutartige tumorartige Läsionen}

Am häufigsten ist die diffuse Variante des tenosynovialen Riesenzelltumors (frühere Bezeichnung: pigmentierte villonoduläre Synovialitis, PVNS) anzutreffen [16]. Arthroskopisch zeigt sich eine villös oder nodulär umgebaute, sandfarbene bis bräunliche Synovialmembran, welche aus klinischer Sicht Ähnlichkeit mit einer rheumatoiden Synovialitis zeigen kann. Histologisch ist das synoviale Stroma dicht angefüllt von Makrophagen und multinukleären Riesenzellen, die in einer Eisenfärbung ausgeprägte Hämosiderindepositionen erkennen lassen.

Andere gutartige Tumoren, die gelegentlich in der Synovialmembran vorkommen, sind die synoviale Chondromatose, das Lipoma arborescens sowie Hämangiome [12]. Auch die heterogene Gruppe der Speichererkrankungen (z. B. M. Fabry, M. Farber, M. Gaucher, Mukopolysaccharidosen) manifestiert sich in der Synovialmembran. Bei diesen Erkrankungen findet sich eine Vermehrung von Makrophagen, Schaumzellen oder Fibroblasten [8].

\section{Kristallarthropathien}

Die kristallinduzierten Erkrankungen können klinisch sowohl unter einem akut-entzündlichen als auch unter einem chronischen Bild auftreten. Histologisch zeichnen sie sich durch Kristalldepositionen und einen unterschiedlich stark ausgeprägten Entzündungsbefund aus. Bei der Arthritis urica wird die Läsion durch Präzipitate von Natriumurat ausgelöst. Diese sind histologisch nur dann erkennbar, wenn die Probe in Alkohol fixiert wur- de, da wässrige Medien die Uratkristalle herauslösen. Es handelt sich um nadelförmige Kristalle, die charakteristischerweise zu Büscheln, aber auch zu dichten rundlichen Aggregaten gelagert sind und eine starke Doppelbrechung im polarisierten Licht aufweisen [14]. Die Kristalle bzw. Kristalllücken sind von Fremdkörperriesenzellen, Makrophagen und Fibroblasten umgeben. Je nach Schweregrad der Entzündung kommen Lymphozyten und neutrophile Granulozyten dazu, und die synoviale Deckzellschicht ist hyperplastisch und fibrinbelegt (• Abb. 2 a).

Die so genannte Pseudogicht oder Chondrokalzinose wird durch Depositionen von Kalziumpyrophosphatdihydrat (KPPD) hervorgerufen und lässt sich von der Arthritis urica unterscheiden, da die Kristalle nicht nadelförmig, sondern feingranulär und zu rundlichen Aggregaten gelagert sind. Diese zeigen in der HE-Färbung eine leichte Basophilie und weisen polarisationsoptisch eine geringere Doppelbrechung auf, sofern sie nicht durch Entkalkung oder zu lange Fixierung aus dem Gewebe herausgelöst wurden [3]. Entzündliche Infiltrate durch Lymphozyten oder Granulozyten sind schwächer ausgeprägt als bei der Gicht (- Abb. 2 b).

\section{Granulomatöse Synovialitiden}

Sollten histomorphologisch Granulome nachweisbar sein, so kommen eine Sarkoidose, Tuberkulose, RA und Fremdkörperreaktionen in Betracht. Ein Problem in der Diagnostik der RA ist allerdings, dass in Synovialisbioptaten häufig keine Granulome anzutreffen sind und das Bild uncharakteristisch ist [10]. 
Pathologe 2008 • [Suppl 2] 29:218-222

DOI 10.1007/s00292-008-1036-9

c) Springer Medizin Verlag 2008

\section{Morawietz · G. Fernahl · V. Krenn Differenzialdiagnostik der Synovialitis}

\section{Zusammenfassung}

In der Synovialmembran kann sich eine Vielzahl von Krankheiten unterschiedlichster Genese manifestieren. Neben den eher seltenen Tumoren und Speichererkrankungen und den häufigeren Kristallarthropathien und akuten bakteriellen Entzündungen sind es insbesondere die chronischen uncharakteristischen Synovialitiden, die in der histopathologischen Praxis oft nicht sicher einer definitiven Diagnose zugeordnet werden können.

Dieser Beitrag gibt einen Überblick über die Differenzialdiagnosen der Gelenkerkrankungen und erläutert den so genannten Synovialitis-Score, der eine Möglichkeit darstellt, chronische uncharakteristische Synovialitiden mit hoher Wahrscheinlichkeit (Sensitivität $60,5 \%$ und Spezifität 95,5\%) dem degenerativen oder dem rheumatischen Formenkreis zuzuordnen.

Schlüsselwörter

Synovialitis · Arthritis · Arthrose · Histopathologie $\cdot$ Synovialitis-Score

\section{Differential diagnosis of synovitis}

\section{Abstract}

The synovial membrane is a site where many diseases with different etiologies can become manifest. Tumors and storage diseases are some of the rare conditions, whereas crystal deposition diseases, acute bacterial infections and in particular chronic uncharacteristic synovitis are frequently encountered. The latter present a diagnostic problem, because they can barely be assigned to concrete diagnoses.

This report will give an overview of the differential diagnosis of joint diseases and will focus on the so-called synovitis score as a tool for the systematic evaluation of chronic uncharacteristic synovitis, providing a possibility to differentiate between degenerative and rheumatic synovitis with a specificity of $60.5 \%$ and a sensitivity of $95.5 \%$.

Keywords

Synovitis - Rheumatic disease - Osteoarthritis . Histopathology $\cdot$ Synovitis score
Eine Tuberkulose kann sich auf hämatogenem Wege in der Synovialmembran manifestieren, was bei etwa $2 \%$ der Tuberkulosepatienten vorkommt [17]. Auch nach Tierbissen sowie dem Baden in Flüssen und Seen kommt es zu synovialen Manifestationen einer Mykobakteriose, die in solchen Fällen allerdings weniger die Gelenke, sondern vielmehr die Sehnenscheiden befällt. Histomorphologisch können typische epitheloidzellige Granulome mit zentraler Nekrose und Langhans-Riesenzellen vorkommen. Die Befunde sind jedoch häufig auch weniger deutlich ausgeprägt, und die zentralen Nekrosen können fehlen [9]. Eine Polymerasekettenreaktion- (PCR-)Untersuchung des Paraffinmaterials auf mykobakterielles Genomäquivalent verfügt über eine wesentlich höhere Sensitivität als die Ziehl-NeelsenFärbung und erlaubt außerdem eine Subspezifikation der Mykobakterienstämme, beispielsweise M. tuberculosis, M. bovis oder M. marinum.

Kleine oder abortive Granulome können auch bei der Brucellose auftreten, die durch intrazytoplasmatische gramnegative Stäbchen hervorgerufen wird und klinisch einen akuteren Verlauf als die Tuberkulose zeigt, histologisch aber nicht sicher von dieser zu unterscheiden ist [1].

Die Sarkoidose kann sich gelegentlich in der Synovialmembran manifestieren. Insbesondere im akuten Stadium können arthritische Beschwerden auftreten. Histologisch finden sich epitheloidzellige Granulome mit Riesenzellen ohne zentrale Nekrosen. Es können aber auch uncharakteristische Aggregate von Lymphozyten vorliegen [13].

Traumatisch oder iatrogen in die Gelenkregion eingebrachte Fremdkörper (z. B. Kakteen- oder Seeigelstachel, Nahtmaterial) führen zu Granulomen und lassen sich konventionell-histologisch oder polarisationsoptisch darstellen. Intra- und paraartikuläre Injektionen von Glukokortikoiden werden mit dem Ziel einer akuten Entzündungshemmung bei RA-Patienten gelegentlich durchgeführt und können zu Nekrosen führen, in denen sich Lücken herausgelöster Kortikoidkristalle finden [7].

\section{Floride, eitrige Synovialitis}

Das klinische Bild einer floriden Synovialitis kann zu einer Synovialisbiopsie Anlass geben, da der Verdacht auf einen akuten Schub einer RA bestehen kann und eine bakterielle Arthritis ausgeschlossen bzw. belegt werden soll. Die floride, bakterielle Synovialitis zeichnet sich durch Ansammlung von neutrophilen Granulozyten und Fibrinpräzipitate auf einer teilweise ulzerierten synovialen Deckzellschicht aus. Darunter findet sich ein kapillarreiches Granulationsgewebe, welches ebenfalls von Granulozyten durchsetzt ist oder bei subakutem Verlauf hauptsächlich Lymphozyten und Plasmazellen enthält [9].

\section{Chronische uncharakteristische Synovialitiden}

Die chronischen uncharakteristischen Synovialitiden zeichnen sich unabhängig von der Ätiologie durch 3 wesentliche Kompartimente aus:

1. die Verbreiterung der synovialen Deckzellschicht,

2. eine Aktivierung der residenten Zellen (synoviale Fibroblasten, Kapillaren),

3. ein lymphozytäres, teils auch plasmazelluläres inflammatorisches Infiltrat.

Zur Erhebung des Synovialitis-Scores werden diese 3 Elemente separat semiquantitativ von o (entspricht Normalbefund) bis 3 (entspricht maximaler pathologischer Veränderung) gradiert. Anschließend werden die 3 Punktwerte addiert, wobei o und 1 Punkt einem Normalbefund entsprechen, 2-4 Punkte einer "Low-grade-Synovialitis" und 5-9 Punkte einer "High-grade-Synovialitis“ (• Tab. 1). Angesichts der bekannten Heterogenität sollte der Score in dem Areal angewandt werden, in dem die Entzündung am deutlichsten ausgeprägt ist. Die Anzahl der Zelllagen der Deckzellschicht sollte an 10 Lokalisationen ausgezählt und das arithmetische Mittel mit einer Nachkommastelle bestimmt werden [10].

Zur Validierung dieses Scores wurde eine Untersuchung an 559 Synovialektomiepräparaten und 59 Biopsaten durchgeführt und mit den jeweiligen klinischen 
Diagnosen korreliert. Hierbei ergaben sich für die einzelnen klinischen Diagnosen folgende Medianwerte (arithmetisches Mittel) der Synovialitis-Scores:

- Kontrollproben: $1(0,72)$,

- Arthrose: 2 (2,33),

- posttraumatische Arthropathie: 2 $(2,06)$,

- Psoriasisarthritis: $3(3,58)$,

- reaktive Arthritis: $5(5,22)$ und

- RA: $5(4,85)$.

Die Unterschiede in den SynovialitisScore-Werten zwischen den degenerativen und den rheumatischen Erkrankungen waren signifikant, und der Befund einer High-grade-Synovialitis als diagnostisches Kriterium für eine rheumatische Erkrankung besaß eine Sensitivität von $60,5 \%$ und eine Spezifität von $95,5 \%$ [5, 6]. Innerhalb der High-grade-Synovialitiden ist eine sichere Unterscheidung zwischen Psoriasisarthritis, reaktiver Arthritis und RA nicht möglich, es sei denn, es liegen rheumatoide Granulome vor. Bei den Low-grade-Synovialitiden finden sich häufig avitale Knorpel- und Knochenfragmente im synovialen Stroma, welche von einer Fremdkörperreaktion umgeben sind. Dieser Befund, der im Rahmen der Arthrose oder eines Traumas auftreten kann, wird als Detritussynovialitis bezeichnet.

\section{Beispiel für die Anwendung des Synovialitis-Scores in der Routinediagnostik}

Übersandt wurden mehrere Synovialisbiopsate aus dem rechten Knie eines 22jährigen Mannes. Anamnestisch sei ein Jahr vor der Arthroskopie ein Trauma erfolgt. Der Patient habe jedoch das Gelenk kontinuierlich weiter belastet, da er professionell Sport betreibe. Aufgrund progredienter Schmerzen habe er sich letztlich einer Arthroskopie unterzogen. Klinisch standen angesichts eines eindrucksvollen endoskopischen Befundes die Differenzialdiagnosen RA und PVNS im Vordergrund ( $\bullet$ Abb. 3 a). Histologisch fanden sich eine geringgradige Verbreiterung der synovialen Deckzellschicht (1 Punkt), eine geringe Zunahme der Dichte der residenten Zellen (1 Punkt) sowie ein schütteres lymphozytäres entzündliches

\begin{tabular}{|c|c|}
\hline \multicolumn{2}{|c|}{ Verbreiterung der synovialen Deckzellschicht: } \\
\hline 0 Punkte & Die synoviale Deckzellschicht ist im Mittel bis 1,7 Zelllagen breit \\
\hline 1 Punkt & Die synoviale Deckzellschicht ist im Mittel 1,8-3,5 Zelllagen breit \\
\hline 2 Punkte & Die synoviale Deckzellschicht ist im Mittel 3,6-5,3 Zelllagen breit \\
\hline 3 Punkte & $\begin{array}{l}\text { Die synoviale Deckzellschicht ist mehr als 5,3 Zelllagen breit, oder es findet sich } \\
\text { eine Ulzeration mit Fibrinexsudation }\end{array}$ \\
\hline \multicolumn{2}{|c|}{ Zelldichte des synovialen Stromas: } \\
\hline O Punkte & Das synoviale Stroma zeigt eine normale Zelldichte \\
\hline 1 Punkt & Das synoviale Stroma zeigt eine gering erhöhte Zelldichte \\
\hline 2 Punkte & $\begin{array}{l}\text { Das synoviale Stroma zeigt eine mittelgradig erhöhte Zelldichte, vereinzelt } \\
\text { können multinukleäre Riesenzellen vorkommen }\end{array}$ \\
\hline 3 Punkte & $\begin{array}{l}\text { Das synoviale Stroma zeigt eine hohe Zelldichte, multinukleäre Riesenzellen } \\
\text { kommen vor, evtl. sind rheumatoide Granulome zu erkennen }\end{array}$ \\
\hline \multicolumn{2}{|c|}{ Leukozytäre entzündliche Infiltration: } \\
\hline O Punkte & Kein Nachweis eines entzündlichen Infiltrats \\
\hline 1 Punkt & $\begin{array}{l}\text { Einzelne, meist perivaskulär gelegene Lymphozyten oder Plasmazellen und } \\
\text { kleine Lymphozytenaggregate }\end{array}$ \\
\hline 2 Punkte & Größere Lymphozytenaggregate \\
\hline 3 Punkte & Dichtes konfluentes lymphozytäres Infiltrat oder Lymphfollikel mit Keimzentren \\
\hline \multicolumn{2}{|l|}{ Gradierung: } \\
\hline $\begin{array}{l}\text { Summe } 0 \\
\text { oder } 1\end{array}$ & Keine Synovialitis \\
\hline Summe $2-4$ & Niedriggradige („Low-grade-“) Synovialitis \\
\hline Summe 5-9 & Hochgradige („High-grade-") Synovialitis \\
\hline
\end{tabular}
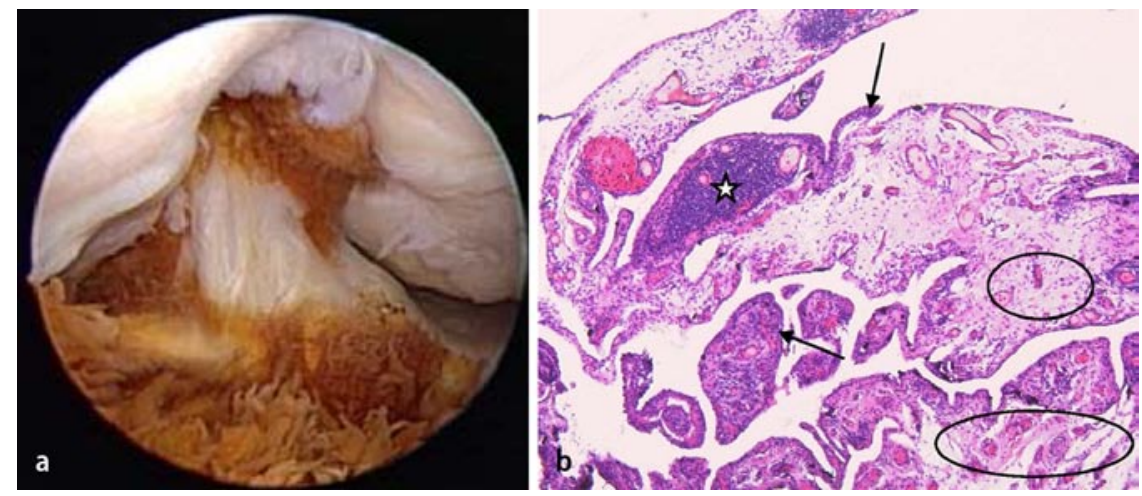

Abb. $3 \Delta$ a Der arthroskopische Befund eines 22-jährigen Sportlers mit Zustand nach Trauma 12 Monate vor der Aufnahme zeigt eine bräunlich verfärbte und villonodulär konfigurierte Synovialmembran. b Histologisch kommen eine geringgradig verbreiterte synoviale Deckzellschicht (1 Punkt, Pfeile), eine diskrete Vermehrung der Kapillaren und Fibroblasten (1 Punkt, Ovale) und ein fokales entzündliches Infiltrat (1 Punkt, Stern) zur Darstellung. Dieser Befund ist am ehesten mit einer posttraumatischen Arthropathie vereinbar (HE-Färbung, Vergr. 50:1)

Infiltrat (1 Punkt, • Abb. 3 b). Der Befund (Score $1+1+1=3$ ) entsprach somit einer Low-grade-Synovialitis und war daher gut mit einer posttraumatischen Arthropathie vereinbar. Eine Erkrankung aus dem rheumatischen Formenkreis war aufgrund des Score-Wertes 5 unwahrscheinlich, und für eine PVNS ergab sich kein Anhalt.

\section{Fazit für die Praxis}

Bei der Beurteilung von Synovialektomiepräparaten und Synovialisbiopsaten sind etliche, teils tumorartige, teils entzündliche Differenzialdiagnosen zu berücksichtigen. Insbesondere für chronische uncharakteristische Synovialitiden ist die Anwendung des SynovialitisScores empfehlenswert, da er eine stan- 
dardisierte Diagnostik ermöglicht und den Befund entweder dem degenerativen (bei einem Score-Wert unter 5 von 9 Punkten) oder dem rheumatischen Formenkreis (5 oder mehr Punkte) zuordnen lässt.

\section{Korrespondenzadresse}

\section{Dr. L. Morawietz}

Institut für Pathologie, Charité-Universitätsmedizin Berlin

Charitéplatz 1, 10117 Berlin

lars.morawietz@charite.de

Danksagung. Diese Arbeit entstand mit freundlicher Unterstützung durch die ENDO-Stiftung - Stiftung des Gemeinnützigen Vereins ENDO-Klinik e.V.

Interessenkonflikt. Der korrespondierende Autor gibt an, dass kein Interessenkonflikt besteht.

\section{Literatur}

1. Andonopoulos AP, Asimakopoulos G, Anastasiou E, Bassaris HP (1986) Brucella arthritis. Scand J Rheumatol 15: 377-380

2. Arnett F, Edworthy S, Bloch D et al. (1988) The American Rheumatism Association 1987 revised criteria for the classification of rheumatoid arthritis. Arthritis Rheum 31: 315-324

3. Chaplin AJ (1976) Calcium pyrophosphate. Histological characterization of crystals in pseudogout. Arch Pathol Lab Med 100: 12-15

4. Kim HJ, Krenn V, Steinhauser G, Berek C (1999) Plasma cell development in synovial germinal centers in patients with rheumatoid and reactive arthritis. J Immunol 162: 3053-3062

5. Krenn V, Morawietz L, Burmester GR, Haupl T (2005) Synovialitis score: histopathological grading system for chronic rheumatic and non-rheumatic synovialitis Z Rheumatol 64: 334-342

6. Krenn V, Morawietz L, Burmester GR et al. (2006) Synovitis-Score: Discrimination between chronic low-grade and high-grade synovitis Histopathology 49: 358-364

7. McCarty DJ, Hogan JM (1964) Inflammatory reaction after intrasynovial injection of microcrystalline adrenocorticosteroid esters. Arthritis Rheum 7: 359-367

8. Mohr W (2000) Stoffwechselkrankheiten. In: Mohr W (Hrsg) Gelenkpathologie. Springer, Berlin Heidelberg New York, S 159-162

9. Mohr W (2000) Entzündungen bekannter Ätiologie. In: Mohr W (Hrsg) Gelenkpathologie. Springer, Berlin Heidelberg New York, S 269-306

10. Morawietz L, Schaeper F, Schroeder JH et al. (2008) Computer-assisted validation of the synovitis score. Virchows Arch 452: 667-673

11. Morawietz L, Classen RA, Schröder JH et al. (2006) Proposal for a histopathological consensus classification of the periprosthetic interface membrane. J Clin Pathol 59: 591-597

12. Nielsen GP, Mandahl N (2002) Lipoma. In: Fletcher CDM, Unni KK, Mertens F (eds) World health organization classification of tumours. Pathology and genetics. Tumours of soft tissue and Bone. IARC, Lyon, pp 20-22
13. Palmer DG, Schumacher HR (1984) Synovitis with non-specific histological changes in synovium in chronic sarcoidosis. Ann Rheum Dis 43: 778-782

14. Rosai J (2004) Bone and joints. In: Rosai J (ed) Rosai and Ackerman's surgical pathology. Mosby, Edinburgh London New York Oxford Philadelphia St Louis Sydney Toronto, pp 2204

15. Schulte E, Fisseler-Eckhoff A, Müller KM (1994) Differentialdiagnose der Synovialitis. Korrelation von arthroskopisch-bioptischen zu klinischen Befunden. Pathologe 15: 22-27

16. Somerhausen NS, Cin PD (2002) Diffuse-type giant cell tumor. In: Fletcher CDM, Unni KK, Mertens $\mathrm{F}$ (eds) World Health Organization Classification of Tumours. Pathology and genetics. Tumours of soft tissue and Bone. IARC, Lyon, pp 112-114

17. Walpert J, Brühl P (1996) Epidemiologie der Skelett- und Urogenitaltuberkulose in Deutschland. Dtsch Ärztebl 93: A469-A470 